Artículo Original

\title{
Impacto de la pandemia COVID-19 en el abordaje del infarto de miocardio con elevación del segmento ST en un centro de referencia del norte del Perú
}

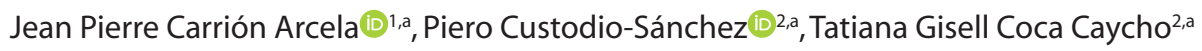

Recibido: 25 de julio de 2021

Aceptado: 14 de septiembre de 2021

Filiación de los autores

Servicio de Cardiología. Hospital Luis Heysen Incháustegui. EsSalud. Chiclayo. Perú.

Servicio de Cardiología. Hospital Nacional Almanzor Aguinaga Asenjo. EsSalud. Chiclayo. Perú. Médico cardiólogo asistente

\section{*Correspondencia}

Jean Pierre Carrión Arcela

$+51914755876$

Correo

jeancarrionarcela@gmail.com

Financiamiento

El presente artículo es autofinan-

ciado.

Conflictos de interés

Los autores de este artículo declara-

mos no tener conflicto interés.

El presente trabajo de investigación ha sido presentado en la Sesión de Temas Libres: XXVIII Congreso Peruano de Cardiología (30 de abril 2021 - Perú

\section{Citar como:}

Carrión Arcela JP, Custodio-Sánchez P, Coca Caycho TG. Impacto de la pandemia COVID-19 en el abordaje del infarto de miocardio con elevación del segmento ST en un centro
de referencia del norte del Perú. Arch Peru Cardiol Cir Cardiovasc. 2021;2(3):159-166. doi: 10.47487/ apcyccv.v2i3.148.

\section{RESUMEN}

Objetivo. Evaluar la repercusión de la pandemia del COVID-19 sobre el abordaje del infarto de miocardio con elevación del segmento ST (IMCEST) en un centro de referencia en el norte del Perú. Métodos. Se realizó un estudio observacional, analítico, tipo cohortes retrospectivas, derivado del registro de síndrome coronario agudo del Hospital Nacional Almanzor Aguinaga Asenjo. Se compararon las características de los pacientes con IMCEST y sus desenlaces a 30 días en dos cohortes según el momento de atención médica: previo a la pandemia o durante pandemia. Resultados. Durante la pandemia del COVID-19 disminuyeron las hospitalizaciones por IMCEST en 53\%; hubo mayor empleo de fibrinólisis en detrimento de la angioplastia primaria, con incrementos en el tiempo de primer contacto médico ( 100 vs. $240 \mathrm{~min}, \mathrm{p}=0,006$ ) y tiempo de isquemia hasta el intervencionismo coronario percutáneo (900 vs. $2880 \mathrm{~min}, \mathrm{p}<0,001$ ). Se encontró mayor frecuencia de falla cardiaca posinfarto $(21,1 \%$ vs. $46,7 \%, p=0,002)$ y menor fracción de eyección del ventrículo izquierdo al alta $(49,2+/-8,6$ vs. $44,8+/-9,3, p=0,009$ ), sin que esto se refleje en un incremento de la mortalidad cardiovascular intrahospitalaria. Conclusiones. La pandemia COVID-19 ha tenido un impacto negativo en el abordaje de pacientes con IMCEST, disminuyó las hospitalizaciones y prolongó los tiempos de reperfusión generando mayor frecuencia de falla cardiaca posinfarto y menor fracción de eyección del ventrículo izquierdo al alta.

Palabras clave: Infarto del miocardio; COVID-19; Reperfusión miocárdica (fuente: DeCS BIREME).

\section{ABSTRACT}

Impact of the COVID-19 pandemic on ST - elevation myocardial infarction management in a reference center of northern Peru

Objective. To evaluate the impact of the COVID-19 pandemic on the ST-Elevation myocardial infarction (STEMI) management in a reference center of northern Peru. Methods. Observational, analytical, retrospective cohort-type study, derivated from the Acute Coronary Syndrome registry of the Almanzor Aguinaga Asenjo National Hospital. The characteristics of the patients with STEMI and their 30-day outcomes were compared in 02 cohorts according to the time of medical care: prior to the pandemic or during the pandemic. Results. During the COVID-19 pandemic, hospitalizations for STEMI decreased by $53 \%$, there was a greater use of fibrinolysis to the detriment of primary angioplasty, with increases in the time of first medical contact ( 100 vs. 240 minutes, $p=0.006$ ) and ischemic time to percutaneous coronary intervention ( 900 vs. 2880 minutes, $p<0.001$ ). This generated a higher frequency of post-infarction heart failure $(21.1 \%$ vs. $46.7 \%, p=0.002)$ and a lower left ventricular ejection fraction at discharge $(49.2+/-$ 8.6 vs $44.8+/-9.3, p=0.009$ ), without an increase in in-hospital cardiovascular mortality. Conclusions. The COVID-19 has had a negative impact on the treatment of patients with STEMI. We found less hospitalizations, prolonged reperfusion times, and higher frequency of post-infarction heart failure and lower left ventricular ejection fraction at discharge. 


\section{Introducción}

La pandemia del COVID-19 generó cambios tanto en la atención de los servicios médicos hospitalarios, que dirigieron la mayor parte de sus esfuerzos hacia la atención de esta nueva enfermedad, como en la población, que evitaba acudir a dichos servicios por miedo al contagio. En este contexto, se evidenció en múltiples centros y países, una disminución en el número de hospitalizaciones por infarto de miocardio con elevación del segmento ST (IMCEST). Tomasoni et al. reportaron una reducción del 33\% en Italia ${ }^{(1)}$; Rodriguez et al. reportó un descenso del 27,6\% en España ${ }^{(2)}$ y Custodio et al. reportaron una reducción del 59\% (3) en el Perú; asimismo, se han reportado menores tasas de reperfusión, menor frecuencia de intervencionismo coronario percutáneo (ICP) primario, y un incremento del tiempo primer contacto médico y del tiempo total isquemia ${ }^{(4)}$. Esto se ha asociado con un incremento en la mortalidad y complicaciones posinfarto en estos pacientes ${ }^{(5,6)}$.

Por lo anteriormente expuesto, y el impacto que esta pandemia ha tenido en la realidad peruana, donde la tasa de no reperfusión es alta y generalmente con tiempos de puerta - balón alejado de estándares internacionales ${ }^{(7,8)}$, nos planteamos como objetivo evaluar la repercusión de la pandemia COVID-19 sobre las estrategias de reperfusión en infarto agudo de miocardio con elevación del Segmento ST en un centro de referencia del norte del Perú.

\section{Materiales y métodos}

Estudio observacional analítico de cohortes retrospectivas. Los pacientes fueron seleccionados del registro de síndrome coronario agudo del Hospital Nacional Almanzor Aguinaga Asenjo, centro de referencia de la macrorregión norte (Lambayeque, Piura, Cajamarca, Tumbes y Amazonas) del Perú. Se realizó un muestreo censal y se compararon las características de los pacientes con IMCEST y sus desenlaces a 30 días, entre dos grupos: abril diciembre 2019 (previo a la pandemia) y abril - diciembre del 2020 (durante la pandemia). Los criterios de inclusión fueron: mayores de 18 años, de ambos sexos, con clínica de angina y/o equivalente anginoso asociado con elevación persistente del segmento ST $\geq$ 2,5 mm en los varones menores de 40 años; $\geq 2 \mathrm{~mm}$ en los varones de 40 o más, $0 \geq 1,5 \mathrm{~mm}$ en las mujeres en las derivaciones $\mathrm{V} 2-\mathrm{V} 3, \mathrm{o}$ $\geq 1 \mathrm{~mm}$ en otras derivaciones ${ }^{(7)}$, independientemente del tiempo de evolución, o con bloqueo completo de rama izquierda del haz de His de novo, se excluyó a pacientes con diagnóstico de infarto agudo de miocardio sin lesiones coronarias, disección coronaria o embolismo coronario.

Se evaluaron y compararon entre ambos grupos: las características sociodemográficas, antecedentes cardiovasculares, estrategias de reperfusión, tiempos en la terapia de reperfusión, complicaciones intrahospitalarias y desenlaces a los 30 días.

Para el análisis estadístico se utilizaron medidas de tendencia central y sus respectivas medidas de dispersión, la prueba T de Student, Chi-cuadradoy el rango de Wilcoxon, según correspondía. Se consideró estadísticamente significativo un valor de $\mathrm{p}$ de $<0,05$. Todos los análisis estadísticos se realizarán a través del programa SPSS versión 25.

\section{Resultados}

El estudio incluyó 96 pacientes con diagnóstico de IMCEST durante los meses de abril-diciembre 2019 y 45 pacientes durante los meses de abril-diciembre 2020. Se encontró una disminución de $53 \%$ en el número total de hospitalizaciones entre ambos períodos (Figura 1).

El sexo masculino predominó en ambos grupos, los factores de riesgo cardiovascular más frecuentes fueron la hipertensión arterial, diabetes mellitus y la dislipidemia, no se encontraron diferencias significativas entre los grupos. La angina típica fue el síntoma de ingreso más frecuente en ambos grupos (90\%), seguida por la disnea; la angina atípica fue proporcionalmente más frecuente durante el período de pandemia $(p=0,019)$, y la localización de infarto más frecuente fue el de cara anterior, seguido del de cara inferior (Tabla 1).

Respecto a las terapias de reperfusión se observó que cerca del $50 \%$ de pacientes no accedió a una terapia de



Figura 1. Hospitalizaciones por IMCEST antes y durante la pandemia del COVID-19 
Tabla 1. Características de los pacientes con IMCEST antes y durante la pandemia por COVID-19

\begin{tabular}{|c|c|c|c|}
\hline Características basales & $\begin{array}{c}\text { Pre-COVID } \\
(\mathrm{N}=96)\end{array}$ & $\begin{array}{l}\text { COVID } \\
(\mathrm{N}=45)\end{array}$ & $\mathbf{p}$ \\
\hline Edad (media $\pm \mathrm{DE})$ & $64,68( \pm 11,4)$ & $66,09( \pm 13,8)$ & 0,551 \\
\hline Sexo masculino & $82(85,4 \%)$ & $36(80 \%)$ & 0,417 \\
\hline \multicolumn{4}{|l|}{ Factores de riesgo } \\
\hline Hipertensión arterial & $59(61,5 \%)$ & $26(57,8 \%)$ & 0,677 \\
\hline Diabetes mellitus & $30(31,3 \%)$ & $14(31,1 \%)$ & 0,987 \\
\hline Dislipidemia & $41(42,7 \%)$ & $14(31,1 \%)$ & 0,188 \\
\hline Enfermedad renal crónica & $13(13,5 \%)$ & $5(11,1 \%)$ & 0,687 \\
\hline \multicolumn{4}{|l|}{ Clínica inicial } \\
\hline Angina típica & $92(95,8 \%)$ & $40(88,9 \%)$ & 0,116 \\
\hline Dolor torácico atípico & $4(4,2 \%)$ & $7(20 \%)$ & 0,019 \\
\hline Disnea & $23(23,96 \%)$ & $13(28,9 \%)$ & 0,531 \\
\hline Arresto cardiaco & $1(1,04 \%)$ & $1(2,22 \%)$ & 0,581 \\
\hline Síncope & $2(2,08 \%)$ & $4(8,88 \%)$ & 0,062 \\
\hline \multicolumn{4}{|l|}{ Ritmo de presentación } \\
\hline Sinusal & $92(95.8 \%)$ & $44(97.78 \%)$ & \multirow{4}{*}{0,813} \\
\hline Fibrilación auricular & $1(1.05 \%)$ & 0 & \\
\hline BAV & $2(2.10 \%)$ & $1(2.22 \%)$ & \\
\hline BCRIHH & $1(1.05 \%)$ & 0 & \\
\hline \multicolumn{4}{|l|}{ Killip Kimbal de ingreso } \\
\hline I & $66(68,7 \%)$ & $27(60 \%)$ & \multirow{4}{*}{0,489} \\
\hline$\|$ & $16(16,7 \%)$ & $12(26,6 \%)$ & \\
\hline III & $7(7,3 \%)$ & $3(6,7 \%)$ & \\
\hline IV & $7(7,3 \%)$ & $3(6,7 \%)$ & \\
\hline \multicolumn{3}{|l|}{ Localización del infarto } & \multirow{5}{*}{0,030} \\
\hline Anterior & $44(45,8 \%)$ & $28(62,2 \%)$ & \\
\hline Inferior & $43(44,8 \%)$ & $12(26,7 \%)$ & \\
\hline Inferoposterior & $9(9,4 \%)$ & $3(6,7 \%)$ & \\
\hline Lateral & 0 & $2(4,4 \%)$ & \\
\hline
\end{tabular}

BCRIHH: bloqueo completo rama izquierda del haz de Hiss.

reperfusión oportuna ( $<12 \mathrm{~h}$ del inicio de síntomas) antes y durante la pandemia COVID-19, con un porcentaje ligeramente mayor en este último período sin significancia estadística. La fibrinólisis fue empleada en mayor proporción durante el período de pandemia con una tasa de éxito del $80 \%$, con disminución del uso de angioplastia primaria, sin alcanzar una diferencia estadísticamente significativa entre ambos grupos (Tabla 2).

Aquellos pacientes que se sometieron a revascularización percutánea presentaron un flujo final TIMI 3 en más del $90 \%$ de casos, la lesión de la arteria descendente anterior fue la causante de infarto en la mayor parte en ambos grupos, pero hubo una mayor frecuencia de enfermedad arterial multivaso durante el período de pandemia, con una diferencia estadísticamente
Tabla 2. Estrategias de reperfusión

\begin{tabular}{lccc}
\hline & $\begin{array}{c}\text { Pre - COVID } \\
\mathbf{( N = 9 6 )}\end{array}$ & $\begin{array}{c}\text { COVID } \\
\mathbf{( N = 4 5 )}\end{array}$ & p \\
\hline Fibrinólisis & $33(34,38 \%)$ & $19(42,2 \%)$ & 0,915 \\
$\quad$ Exitosa & $23(69,7 \%)$ & $15(80 \%)$ & 0,368 \\
Coronariografía & $87(90,6 \%)$ & $41(91,1 \%)$ & 0,926 \\
ICP & & & \\
$\quad$ Primaria & $15(15,6 \%)$ & $3(6,7 \%)$ & 0,137 \\
$\quad$ Farmacoinvasiva & $19(19,8 \%)$ & $11(24,4 \%)$ & 0,622 \\
\hline
\end{tabular}

ICP: intervención coronaria percutánea. 
significativa ( $92 \%$ vs. $58 \%, p=0,021)$. Asimismo, estas lesiones coronarias no responsables, fueron tratadas con mayor frecuencia durante la misma hospitalización durante el periodo de pandemia $(p=0,016)$ (Tabla 3$)$.

Uno de los hallazgos más importantes fue la prolongación de los tiempos de reperfusión, así el tiempo de isquemia a intervención coronaria percutánea (ICP) fue más largo durante la pandemia ( 2880 frente a 900 min, $p<0,001$ ), siendo este incremento una sumatoria entre una mayor demora al primer contacto médico (240 frente a $100 \mathrm{~min}, \mathrm{p}=0,006$ ) y un incremento del tiempo puerta - balón (945 frente a 610 min, p=0,073) (Figura 2).

La terapia farmacológica empleada en ambos grupos fue similar, la complicación intrahospitalaria más frecuente fue la falla cardiaca, aunque con mayor frecuencia de presentación durante el período del COVID-19, con diferencia estadísticamente significativa $(46,7 \%$ frente a $21,1 \%, p=0,002)$;adicionalmente, la fracción de eyección al alta fue menor durante la pandemia $(49,2$ +/- 8,6 vs. 44,8 +/- 9,3, $\mathrm{p}=0,009$ ) (Tabla 4).

Estos hallazgos no se relacionaron con un impacto en mortalidad cardiovascular intrahospitalaria ni a los 30 días. Finalmente, cabe destacar que en la cohorte durante la pandemia, diez pacientes fueron diagnosticados con COVID-19 y durante su evolución dos fallecieron a los 30 días, lo que incrementó la mortalidad por causas no cardiovasculares $(p=0,046)$ (Tabla 4).

\section{Discusión}

Nuestro trabajo evaluó el impacto de la pandemia COVID-19 en el manejo del IMCEST en la macrorregión norte del Perú, evidenciando que el mismo fue negativo, disminuyendo el número de hospitalizaciones en más del $50 \%$ retrasando e incluso disminuyendo la terapia de reperfusión con el consiguiente incremento de complicaciones agudas como falla cardiaca y una menor fracción de eyección al alta (Figura 3).

La disminución en el número de ingresos hospitalarios por IMCEST ha sido una constante en los diferentes estudios con una variación entre $20-60 \%{ }^{(2,3)}$; de acuerdo con esto, nuestro estudio muestra también una reducción importante de hospitalizaciones en el norte del país. Esto puede ser motivado por diferentes razones, entre las que Perrin Nils et al. destacan el temor al contagio o propagación del COVID-19 luego del ingreso hospitalario o el no querer agregar una carga al sistema de salud ${ }^{(9)}$. Además, debemos tomar en cuenta la posibilidad de infradiagnóstico del síndrome coronario agudo ${ }^{(10)} y$ el incremento de mortalidad extrahospitalaria, secundaria a un retraso en la atención oportuna durante la pandemia ${ }^{(11)}$.

Por otro lado, aunque el tiempo es considerado el mayor limitante para lograr un resultado óptimo en la terapia

Tabla 3. Características de la coronariografía en ambos grupos

\begin{tabular}{|c|c|c|c|}
\hline & $\begin{array}{c}\text { Pre-COVID } \\
(\mathrm{N}=34)\end{array}$ & $\begin{array}{l}\text { COVID } \\
(\mathrm{N}=14)\end{array}$ & $\mathbf{p}$ \\
\hline \multicolumn{4}{|l|}{ Arteria responsable del infarto } \\
\hline A. descendente anterior & $18(52,9 \%)$ & $9(64,3 \%)$ & \\
\hline A. coronaria derecha & $5(14,7 \%)$ & 0 & 0,314 \\
\hline A. circunfleja & $11(32,4 \%)$ & $5(35,7 \%)$ & \\
\hline \multicolumn{4}{|l|}{ TIMI inicial } \\
\hline TIMI 0 & $13(38,2 \%)$ & $1(7,1 \%)$ & \\
\hline TIMI 1 & $8(23,5 \%)$ & $1(7,1 \%)$ & \\
\hline TIMI 2 & $11(32,4 \%)$ & $11(78,6 \%)$ & 0,025 \\
\hline TIMI 3 & $2(5,9 \%)$ & $1(7,1 \%)$ & \\
\hline \multicolumn{4}{|l|}{ TIMI final } \\
\hline TIMI 0 & $0(0,0 \%)$ & $0(0,0 \%)$ & \\
\hline TIMI 1 & $1(2,9 \%)$ & $0(0,0 \%)$ & 0,803 \\
\hline TIMI 2 & $2(5,9 \%)$ & $1(7,1 \%)$ & \\
\hline TIMI 3 & $31(91,2 \%)$ & $13(92,9 \%)$ & \\
\hline Enfermedad multivaso & $20(58,82 \%)$ & $13(92,85 \%)$ & 0,021 \\
\hline ICP de ARNI & $4(11,76 \%)$ & $6(42,85 \%)$ & 0,016 \\
\hline
\end{tabular}

ICP: intervención coronaria percutánea. ARNI: arterias no responsables del infarto. 


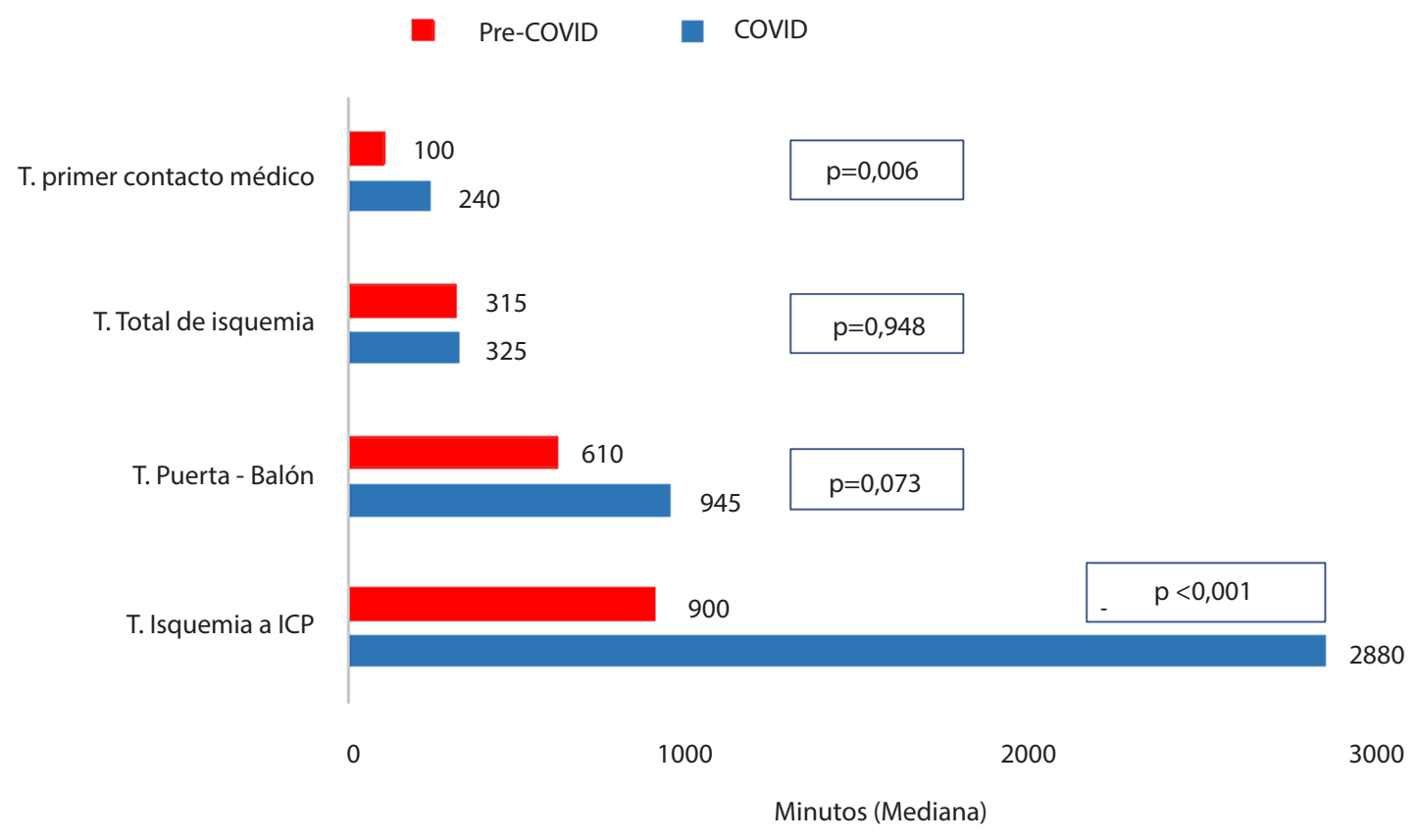

Figura 2. Tiempos de reperfusión antes y durante la pandemia del COVID-19

ICP: ilntervención coronaria percutánea

Tabla 4. Terapia farmacológica, complicaciones intrahospitalarias y desenlaces a 30 días

\begin{tabular}{|c|c|c|c|}
\hline & $\begin{array}{c}\text { Pre-COVID } \\
(\mathrm{N}=96)\end{array}$ & $\begin{array}{l}\text { COVID } \\
(\mathrm{N}=45)\end{array}$ & $\mathbf{p}$ \\
\hline \multicolumn{4}{|l|}{ Terapia farmacológica } \\
\hline Vasopresores & $15(15,6 \%)$ & $(17,8 \%)$ & 0,747 \\
\hline Inotrópicos & $20(20,8 \%)$ & $15(33,3 \%)$ & 0,109 \\
\hline Nitratos & $39(40,6 \%)$ & $19(42,2 \%)$ & 0,857 \\
\hline \multicolumn{4}{|c|}{ Complicaciones intrahospitalarias } \\
\hline Falla cardiaca & $20(21,1 \%)$ & $21(46,7 \%)$ & 0,002 \\
\hline Shock cardiogénico & $16(16,7 \%)$ & $7(15,6 \%)$ & 0,868 \\
\hline ECV & $1(1,1 \%)$ & $0(0 \%)$ & 0,490 \\
\hline Sangrado mayor & $3(3,2 \%)$ & $0(0 \%)$ & 0,228 \\
\hline Parada cardiaca & $5(5,2 \%)$ & $6(13,3 \%)$ & 0,094 \\
\hline Muerte cardiovascular & $12(12,5 \%)$ & $3(6,7 \%)$ & 0,295 \\
\hline Muerte no cardiovascular & $3(3,2 \%)$ & $0(0 \%)$ & 0,226 \\
\hline FEVI al alta & $49,23( \pm 8,59)$ & $44,77( \pm 9,28)$ & 0,009 \\
\hline \multicolumn{4}{|l|}{ Desenlaces a 30 días } \\
\hline Rehospitalización & $6(7,3 \%)$ & $2(4,8 \%)$ & 0,584 \\
\hline Reinfarto & $1(1,2 \%)$ & 0 & 0,472 \\
\hline Muerte cardiaca & 0 & 0 & \\
\hline Muerte no cardiaca & 0 & $2(4,8 \%)$ & 0,046 \\
\hline
\end{tabular}


Figura 3. Imagen central: Impacto de la pandemia COVID-19 sobre el abordaje del infarto de miocardio con elevación del segmento ST

\section{Objetivos}

Evaluar la repercusión de la pandemia COVID-19 en el abordaje del IMCEST en un centro de referencia en el norte del Perú (Hospital Nacional Almanzor Aguinaga Asenjo, Chiclayo, Perú.)

\section{Diseño del Estudio}

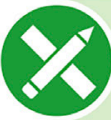

Cohorte retrospectiva

Pacientes con IMCEST
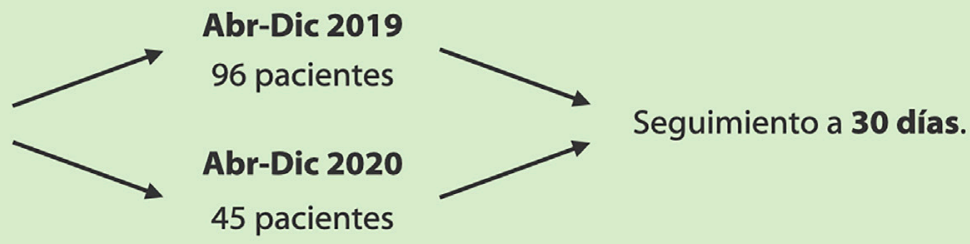

\section{Resultados}

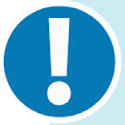

Mayor tiempo a primer contacto médico

Mayor tiempo a $\mathrm{PCl}$

Mayor incidencia de falla postinfarto

$$
\begin{array}{rlrl}
100 & >240 \mathrm{~min} & & (p=0.006) \\
900 & >2880 \mathrm{~min} & & (p<0.001) \\
21.1 \% & >46.7 \% & & (p=0.002) \\
49.2 \%(+-8.6) & >44.8 \%(+-9.3) & (p=0.009)
\end{array}
$$$$
\text { Menor FEVI al alta }
$$

No cambio aparente en mortalidad hospitalaria

\section{Conclusiones}

La pandemia COVID-19 ha tenido un impacto negativo en el manejo y evolución de los pacientes con IMCEST destacando un mayor tiempo de isquemia y mayor falla postinfarto.

Carrión Arcela JP et al. Arch Peru Cardiol Cir Cardiovasc. 2021;2(3):159-166

de reperfusión ${ }^{(12,13)}$; en nuestro país aún estamos alejados de las recomendaciones internacionales, ya que incluso antes de pandemia los tiempos alcanzados eran mucho mayores que los objetivos. La pandemia empeoró esta situación incrementando no solo el tiempo primer contacto médico, sino también el tiempo de isquemia a ICP y tiempo de isquemia total. Las probables causas para explicar estos hallazgos se encuentran en las medidas sanitarias tomadas por el estado, las cuales retrasaban el traslado de paciente dentro o fuera de la región; la información brindada sobre el manejo de la pandemia en los hospitales fortalecían el temor al contagio durante la hospitalización; la falta de espacios en emergencia para la atención de urgencias cardiovasculares; la falta de fibrinolíticos, y el tiempo dedicado a las medidas de protección requeridas previa a la realización de los procedimientos. Esto limitó el acceso a la terapia de reperfusión incrementando la posibilidad de no reperfusión. El presente trabajo muestra una proporción de $50 \%$ de pacientes no reperfundidos, valor mayor a lo reportado en nuestro país hace unos años (33\%); esto muestra la realidad nacional fuera de la capital (8), cifras que son más elevadas en los departamentos más alejados y en centros sin insumos ni capacidad de referencia oportuna. Esto da una idea de la fragilidad que tiene la organización de nuestro sistema de salud, que no logró adaptarse a este escenario adverso descuidando el abordaje de esta y otras patologías cardiovasculares de alta morbimortalidad ${ }^{(14)}$.

La finalidad del manejo de IMCEST es lograr la reperfusión en el menor tiempo posible siguiendo la premisa «tiempo es músculo» ${ }^{(15)}$. En los inicios de la pandemia desde China ${ }^{(16)}$ se motivó un mayor empleo de la fibrinólisis en detrimento de la angioplastia primaria como la primera estrategia de reperfusión, dada su mayor viabilidad con relación a la infraestructura y equipos necesarios, más aun en ese contexto donde se exigía descartar el diagnóstico de infección por SARS-COV-2 y un equipo de bioseguridad acorde como requisito de ingreso a sala de 
hemodinámica; sin embargo, posteriormente el consenso de la Sociedad de Angiografía e Intervenciones Cardiovasculares (SCAI) y el Colegio Americano de Cardiología (ACC) ${ }^{(17)}$ reafirmaron la angioplastia primaria como la primera estrategia de reperfusión a elegir, siempre que se realice en los tiempos adecuados, pues su empleo genera menores tasa de mortalidad, reinfarto y accidente cerebrovascular tanto a corto como largo plazo ${ }^{(18,19)}$. A pesar de estas recomendaciones, así como nuestro estudio mostró un descenso en la realización de angioplastias primarias, el registro ISACS-STEMI COVID en Italia mostró también una reducción del $19 \%$ de angioplastias primarias ${ }^{(20)}$; Kwok et al. reportaron que en Inglaterra hubo una reducción del 43\% de angioplastias en comparación con los promedios mensuales previos ${ }^{(21)}$.

Es importante resaltar que los pacientes que ingresaron durante la pandemia COVID-19 mostraron una frecuencia mayor de enfermedad multivaso, hallazgo también reportado en otros estudios como en el de Rodríguez-Leor et al. ${ }^{(2)}$. Una posible explicación a esto es que los pacientes que acudían a recibir atención médica eran aquellos que presentaban mayor carga isquémica. El abordaje brindado a este tipo de pacientes fue el más adecuado, pues un gran porcentaje recibió revascularización completa antes del alta.

Las consecuencias de los retrasos y la disminución de las terapias de reperfusión durante la pandemia COVID-19 se ven reflejadas en una mayor frecuencia de presentación de insuficiencia cardiaca y una menor fracción de eyección del ventrículo izquierdo al alta, lo cual se correlacionan con los resultados obtenidos por Primessnig et al. en Alemania ${ }^{(21)}$ y De Rosa et al. en Italia ${ }^{(4)}$.
Finalmente, el impacto negativo generado por la pandemia COVID-19 continúo incluso en el seguimiento a los 30 días, pues incrementó la mortalidad por todas las causas, a expensas principalmente de las causas no cardiovasculares, esto debido a factores de mortalidad inherentes a pacientes diagnosticados con COVID-19 como edad > 55 años, hipertensión arterial, diabetes mellitus, entre otros ${ }^{(23,24)}$.

\section{Limitaciones}

A pesar de ser un hospital de referencia de la macrorregión norte del Perú nuestro tamaño muestral es pequeño en relación a otros trabajos presentados previamente, y al ser un estudio regional con características sociodemográficas y territoriales propias no permite conocer la realidad nacional en su totalidad.

\section{Conclusiones}

La pandemia COVID-19 generó en la población estudiada: un menor número de hospitalizaciones, retrasos en el tiempo de reperfusión; mayor frecuencia de falla cardiaca pos-infarto; una menor fracción de eyección del ventrículo izquierdo y mayor mortalidad por todas las causas en los 30 primeros días después del alta. Estos resultados deben llamar la atención de nuestras autoridades sanitarias para fortalecer y priorizar la atención médica oportuna del infarto agudo de miocardio, con el fin de lograr mejores resultados a corto y largo plazo.

\section{Contribución de autores}

Los autores del presente caso declaran haber participado íntegramente en la elaboración, revisión y redacción del presente artículo científico.

\section{Referencias bibliográficas}

1. Tomasoni D, Adamo M, Italia L, Branca L, Chizzola G, Fiorina C, et al. Impact of COVID-2019 outbreak on prevalence, clinical presentation and outcomes of ST-elevation myocardial infarction. J Cardiovasc Med (Hagerstown). 2020 Nov; 21(11):874-81. doi: 10.2459/ JCM.0000000000001098.

2. Rodríguez-Leor O, Cid-Álvarez B, Pérez de Prado A, RosselloX, Ojeda S, Serrador A, et al. Impacto de la COVID-19 en el tratamiento del infarto agudo de miocardio con elevación del segmento ST. La experiencia española [Impact of COVID-19 on ST-segment elevation myocardial infarction care. The Spanish experience]. Rev Esp Cardiol. 2020 Dec;73(12):994-1002. Spanish. doi: 10.1016/j.recesp.2020.07.033.

3. Custodio - Sánchez P, Miranda D, Murillo L. Impacto de la pandemia por COVID-19 sobre la atención del infarto de miocardio ST elevado en el Perú. Arch Peru Cardiol Cir Cardiovasc [Internet]. 9 de julio de 2020. https://doi.org/10.47487/apcyccv.v1i2.22

4. De Rosa S, Spaccarotella C, Basso C, Calabró MP, Curcio A, Filardi PP, et al. Reduction of hospitalizations for myocardial infarction in Italy in the COVID-19 era. Eur Heart J. 2020 Jun 7;41(22):2083-2088. doi: 10.1093/eurheartj/ehaa409.

5. Stefanini GG, Montorfano M, Trabattoni D, Andreini D, Ferrante G, Ancona M, et al. ST-Elevation Myocardial Infarction in Patients
With COVID-19. Circulation. 2020; 141:2113-16. DOI: 10.1161/ CIRCULATIONAHA.120.047525

6. Choudry FA, Hamshere SM, Rathod KS, Akhtar MM, Archbold RA, Guttmann OP, et al. High thrombus burden in patients with COVID19 presenting with ST-segment elevation myocardial infarction. J Am Coll Cardiol 2020; 76:1168-76. doi: 10.1016/j.jacc.2020.07.022.

7. Ibáñez B, James S, Agewall S, Antunes MJ, Bucciarelli-Ducci C, Bueno H, et al. Guía ESC 2017 sobre el tratamiento del infarto agudo de miocardio en pacientes con elevación del segmento ST. Rev Esp Cardiol 2017; 70(12): 1082.e1-e61. DOI: 10.1016/j.recesp.2017.10.048

8. Chacón-Diaz M, Vega A, Aráoz O, Ríos P, Baltodano R, Villanueva F, et al. Características epidemiológicas del infarto de miocardio con elevación del segmento ST en Perú: resultados del PEruvian Registry of ST-segment Elevation Myocardial Infarction (PERSTEMI). Arch Cardiol Mex. 2018; 88(5):403 - 12. DOI: 10.1016/j.acmx.2017.11.009

9. Perrin N, Iglesias JF, Rey F, Benzakour L, Cimci M, Noble S, et al. Impact of the COVID-19 pandemic on acute coronary syndromes. Swiss Med Wkly. 2020 Dec 31;150:w20448. doi: 10.4414/smw.2020.20448.

10. Gorini F, Chatzianagnostou K, Mazzone A, Bustaffa E, Esposito A, Berti S, et al. "Acute Myocardial Infarction in the Time of COVID-19": A 
Review of Biological, Environmental, and Psychosocial Contributors. Int J Environ Res Public Health. 2020 Oct 9;17(20):7371. doi: 10.3390/ ijerph17207371.

11. Woolf SH, Chapman DA, Sabo RT, Weinberger DM, Hill L. Excess Deaths From COVID-19 and Other Causes, March-April 2020. JAMA. 2020;324:510-3. doi: 10.1001/jama.2020.11787.

12. Sánchez Fernández L, Álvarez Rodríguez J, Casado Flores I, Botas Rodríguez J, de Albas Montero J, de Andrés, et al. Código Infarto Madrid. Madrid. 2013. Disponible en: http://www.somamfyc. com/Portals/0/PropertyAgent/400/Files/416/C\%C3\%B3digo\%20 Infarto\%20DEF.pdf

13. Betriu A, Masotti M. Comparison of mortality rates in acute myocardial infarction treated by percutaneous coronary intervention versus fibrinolysis. Am J Cardiol. 2005 Jan 1;95(1):100-1. doi: 10.1016/j. amjcard.2004.08.069.

14. Gozzer E, Canchihuamán F, Espinoza R. COVID-19 y la necesidad de actuar para mejorar las capacidades del Perú frente a las pandemias. Rev Peru Med Exp Salud Publica. 2020;37(2):371-3. doi: https://doi. org/10.17843/rpmesp.2020.372.5410.

15. Ortigosa Aso J, Silva Melchor L. Estrategias de reperfusión en el infarto agudo. Rev Esp Cardiol. 2008;61(1):6-9. DOI: 10.1157/13114950.

16. Zeng J, Huang J, Pan L. How to balance acute myocardial infarction and COVID-19: the protocols from Sichuan Provincial People's Hospital. Intensive Care Med. 2020 Jun;46(6):1111-3. doi: 10.1007/ s00134-020-05993-9.

17. Mahmud E, Dauerman HL, Welt FGP, Messenger JC, Rao SV, Grines $C$, et al. Management of Acute Myocardial Infarction During the COVID-19 Pandemic. J Am Coll Cardiol 2020; 76: 1375 - 84. DOI: $10.1002 / \mathrm{ccd} .28946$
18. Keeley EC, Boura JA, Grines CL. Primary angioplasty versus intravenous thrombolytic therapy for acute myocardial infarction: a quantitative review of 23 randomised trials. Lancet. 2003;361:13-20. DOI: $10.1016 / 50140-6736(03) 12113-7$

19. Huynh T, Perron S, O'Loughlin J, Joseph L, Labrecque M, Tu JV, et al. Comparison of primary percutaneous coronary intervention and fibrinolytic therapy in ST-segment-elevation myocardial infarction. Bayesian hierarchical meta-analyses of randomized controlled trials and observational studies. Circulation. 2009;119:3101-9. DOI: 10.1161/CIRCULATIONAHA.108.793745

20. De Luca G, Verdoia M, Cercek M, Jensen LO, Vavlukis M, Calmac L, et al. Impact of COVID-19 Pandemic on Mechanical Reperfusion for Patients With STEMI. J Am Coll Cardiol. 2020 Nov 17;76(20):2321-30. doi: 10.1016/j.jacc.2020.09.546.

21. Kwok CS, Gale CP, Kinnaird T, Curzen N, Ludman P Kontopantelis E, et al. Impact of COVID-19 on percutaneous coronary intervention for STelevation myocardial infarction. Heart. 2020 Dec;106(23):1805-11. doi: 10.1136/heartjnl-2020-317650.

22. Uwe Primessnig, Burkert M. Pieske, Mohammad Sherif. Increased mortality and worse cardiac outcome of acute myocardial infarction during the early COVID-19 pandemic. ESC Heart Failure 2021; 8 : 333-43. https://doi.org/10.1002/ehf2.13075

23. Jordan RE, Adab P, Cheng KK. Covid-19: risk factors for severe disease and death. BMJ. 2020 Mar 26;368:m1 198. doi: 10.1136/bmj.m1198.

24. Ortiz-Prado E, Simbaña-Rivera K, Gómez-Barreno L, RubioNeira M, Guaman LP, Kyriakidis NC, et al. Clinical, molecular, and epidemiological characterization of the SARS-CoV-2 virus and the Coronavirus Disease 2019 (COVID-19), a comprehensive literature review. Diagn Microbiol Infect Dis. 2020 Sep;98(1):115094. doi: 10.1016/j.diagmicrobio.2020.115094. 